\title{
К вопросу о моделировании термодинамического
}

\section{взаимодействия частиц, взвешенных в двумерной}

\section{среде}

\section{А. О. Сыромясов, Ю. В. Понкратова, Т. В. Меньшакова}

\section{Национальный исследовательский Мордовский государственный университет} (2. Саранск, Российская Федерачия)

\begin{abstract}
Аннотация. Аналитическое решение задачи о распределении температуры в среде с инородными включениями затруднено в связи со сложностью ее геометрии, поэтому для моделирования термодинамических процессов на микроуровне в дисперсных системах применяются асимптотические или численные методы. Для ответа на вопрос о сходимости и сравнительной точности этих методов авторы рассматривают модельную задачу о двух идентичных круглых частицах в безграничной плоской среде с постоянным на бесконечности перепадом температуры. Полученное ранее мультипольное разложение решения в статье доведено до высоких степеней малого параметра, представляющего собой безразмерный радиус термодинамически взаимодействующих частиц. Описан подход к численному моделированию термодинамического взаимодействия частиц в пакете ANSYS; в частности, изучена проблема выбора приближенных граничных условий. Выявлено, что одним из основных источников погрешности метода конечных элементов является замена безграничной среды расчетной областью конечных размеров. Для определения границ области в задаче со множественными включениями авторами разработан метод фиктивной частицы, согласно которому совокупность инородных тел на больших расстояниях от центра области, занятой ими, может быть приближенно заменена одним эквивалентным телом большего радиуса. На конкретных числовых данных исследована зависимость размеров расчетной области, обеспечивающих приемлемую точность, от сравнительных характеристик несущей среды и инородных тел. Авторами проведен ряд численных экспериментов, в ходе которых подтверждена сходимость методов мультипольного разложения и конечных элементов и близость результатов их работы.

Ключевые слова: термодинамическое взаимодействие, дисперсная среда, уравнение теплопроводности, мультиполь, асимптотическое разложение, метод конечных элементов

Для цитирования: Сыромясов А.О., Понкратова Ю.В., Меньшакова Т. В. К вопросу о моделировании термодинамического взаимодействия частиц, взвешенных в двумерной среде // Журнал Средневолжского математического общества. 2021. Т. 23, № 4. С. 444-460. DOI: https://doi.org/10.15507/2079-6900.23.202104.444-460
\end{abstract}

\section{1. Введение}

Одной из актуальных задач механики гетерогенных сред является нахождение распределения температуры в веществе, содержащем множественные инородные включения. Эта задача может иметь самостоятельное значение: так, осреднение температурного поля позволяет найти эффективные характеристики дисперсных сред [1]. С другой стороны, ее решение может выступать этапом при рассмотрении более сложных проблем. Примером служит моделирование течения жидкости со свойствами, зависящими от температуры, в случае, когда температура среды не постоянна, а сама среда содержит инородные примеси [2;3]. При некоторых условиях удается отделить термодинамическую задачу от гидродинамической, найти распределение температуры в "неподвижной" среде и использовать его при описании течения жидкости [4; 5]. 
Если интерес в задаче представляют коллективные эффекты (иначе говоря, термодинамическое взаимодействие инородных тел), то следует выяснить, как невозмущенное распределение температуры искажается двумя, тремя и т.д. частицами. Для этого предполагается, что в среде находится группа инородных включений, а вдалеке от них температура есть известная функция координат и, быть может, времени. Аналогичный подход развивается, например, в [6;7; 8] при моделировании гидродинамического взаимодействия частиц и определения эффективных характеристик суспензии твердых сфер в вязкой жидкости.

Аналитическое решение задачи об одном шарообразном включении в безграничной среде с постоянным на бесконечности градиентом температуры хорошо известно и имеет простой вид [9]. При рассмотрении инородных тел менее элементарной формы вид решения усложняется. Так, аналогичное с математической точки зрения описание электрического поля в среде с проводящим эллипсоидом требует введения специфической системы координат и привлечения эллиптических интегралов [10]. При моделировании гидродинамического взаимодействия шарообразных частиц взвеси в жидкости может вводиться бисферическая система координат [11].

В целом необходимо отметить, что аналитическое решение уравнения Лапласа при описании систем из нескольких частиц (или даже одной частицы, но несферической формы) в среде с иными свойствами весьма затруднено. Использование специально подобранных систем координат приводит к весьма громоздким выражениям для искомых функций. Кроме того, если требуется описать взаимодействие более чем двух частиц, то подобрать систему координат, в которой бы переменные в уравнении Лапласа разделялись, невозможно. В связи с этим применяются асимптотические разложения решений указанного уравнения по малому параметру [8]. Проблема заключается в том, что асимптотические ряды могут не быть сходящимися, и не всегда удается заранее гарантировать, что они хорошо приближают искомые величины [12].

Альтернативой асимптотическим разложениям служит использование численных методов, например, метода конечных элементов [13]. Однако их применение сталкивается со следующей трудностью: расчетная область имеет конечные размеры, в то время как расчет следует произвести для группы тел в безграничном пространстве. Поэтому возникает вопрос: какими должны быть размеры конечной области, заменяющей исходную бесконечную среду, чтобы возникающая от такой замены погрешность не оказала существенного влияния на результат вычислений.

Соответственно вышеизложенному, авторы статьи ставили перед собой следующие задачи. Во-первых, убедиться в сходимости асимптотических мультипольных разложений, получаемых при моделировании распределения температуры в сплошной среде с включениями. Во-вторых, предложить метод оценки размеров расчетной области при решении указанной задачи методом конечных элементов. В-третьих, сравнить решения этой задачи, полученные асимптотическим и численным методами.

Расчеты производятся на модельном примере: в безграничную плоскую среду помещены две идентичные круглые частицы, причем градиент температуры на бесконечном удалении от них является постоянным вектором.

\section{2. Постановка задачи о термодинамическом взаимодействии двух частиц в плоской безграничной среде}

Предположим, что в неподвижную безграничную плоскую среду с постоянной теплопроводностью $\kappa_{f}$ помещены две одинаковые круглые частицы 1 и 2 , имеющие радиус

А. О. Сыромясов, Ю. В. Понкратова, Т. В. Меньшакова. К вопросу о моделировании ... 
$a$ и теплопроводность $\kappa_{p}$ так, что расстояние между их центрами равно $r$. Распределение температуры в среде и частицах стационарно. Бесконечно далеко от обеих частиц градиент температуры есть известный постоянный вектор, произвольно ориентированный по отношению к прямой, соединяющей центры инородных частиц. На границе этих включений температура и тепловой поток не должны претерпевать разрыв; кроме того, внутри частиц температура должна оставаться конечной.

Введем систему координат $O x_{1} x_{2}$, начало $O$ которой находится в центре круга 1 . Ось $O x_{1}$ направим так, что центр круга 2 окажется в точке, имеющей радиус-вектор $\vec{r}=(r ; 0)$. Координатную ось $O x_{2}$ направим перпендикулярно $O x_{1}$ (рис. 2.1).

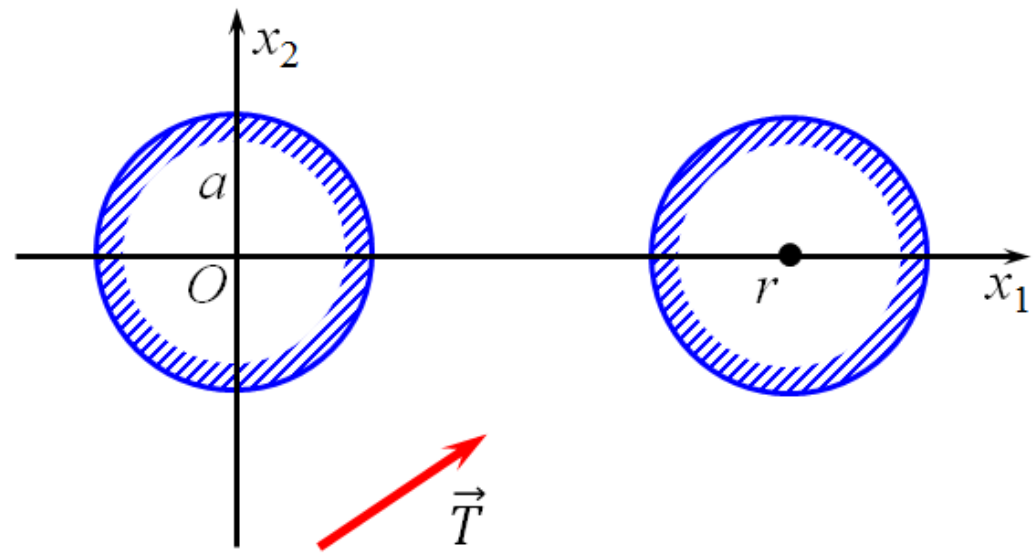

Рис. 2.1. Геометрия задачи

Fig 2.1. Geometry of the problem

Условимся, что вектор $\vec{x}=\left(x_{1}, x_{2}\right)$ задает положение произвольной точки относительно начала координат, а вектор $\vec{y}$ - от от центра того или иного включения.

Тогда стационарность температуры можно записать в форме

$$
\begin{gathered}
\Delta_{\vec{x}} T_{f}=0, \\
\Delta_{\vec{y}} T_{p}(N)=0, \quad N=1,2,
\end{gathered}
$$

где $T_{f}=T_{f}(\vec{x})$ - температура вне частиц, $T_{p}(N)=T_{p}(N, \vec{y})$ - температура внутри $N$-й частицы; $\Delta_{\vec{x}}$ и $\Delta_{\vec{y}}$ суть операторы Лапласа, в которых частные производные берутся по компонентам векторов $\vec{x}$ и $\vec{y}$, соответственно. Далее при возможности векторные индексы у лапласианов будут опускаться.

Условие на удалении от инородных тел имеет вид

$$
\operatorname{grad}_{\vec{x}} T_{f}(\vec{x}) \rightarrow \vec{T}, \quad|\vec{x}| \rightarrow \infty
$$

где $\vec{T}=\left(T_{1}, T_{2}\right)$ - постоянный вектор.

Непрерывность температуры и теплового потока на границе каждого из кругов выражается равенствами ( $\vec{n}$ - единичная внешняя нормаль)

$$
T_{f}=T_{p}(1), \kappa_{f} \frac{\partial T_{f}}{\partial n}=\kappa_{p} \frac{\partial T_{p}(1)}{\partial n}, \quad|\vec{x}|=a,
$$




$$
T_{f}=T_{p}(2), \kappa_{f} \frac{\partial T_{f}}{\partial n}=\kappa_{p} \frac{\partial T_{p}(2)}{\partial n}, \quad|\vec{x}-\vec{r}|=a .
$$

Наконец, обозначим через $T_{0}<\infty$ невозмущенную температуру в центре первого включения. Это позволяет записать поле температуры, не искаженное инородными частицами, в виде

$$
T_{\infty}(\vec{x})=T_{0}+\vec{T} \cdot \vec{x}=T_{0}+T_{1} x_{1}+T_{2} x_{2} .
$$

Соответственно, величина $T^{\prime}$, равная $T_{f}-T_{\infty}$ вне инородных тел и $T_{p}-T_{\infty}$ внутри них, есть возмущение, вносимое ими в исходное распределение температуры.

Далее величина $T_{0}$ не играет существенной роли и потому считается равной нулю.

Суммируя вышеизложенное, получим, что необходимо найти функции $T_{f}, T_{p}(1)$ и $T_{p}(2)$, удовлетворяющие уравнениям (2.1), (2.2), условию на бесконечности (2.3) и граничным условиям (2.4), (2.5).

\section{3. Мультипольное решение задачи}

\section{1. Описание метода}

Применительно к плоской задаче о термодинамическом взаимодействии частиц метод мультипольного разложения был описан в [14] и вкратце заключается в следующем.

Поскольку искомые $T_{f}$ и $T_{p}(N), N=1,2$, суть гармонические функции, они записываются в виде рядов мультиполей - частных производных фундаментального решения $L_{0}$ уравнения Лапласа на плоскости:

$$
L_{0}(\vec{x})=\ln |\vec{x}|, \quad L_{j \ldots k}(\vec{x})=\frac{\partial}{\partial x_{j}} \cdots \frac{\partial}{\partial x_{k}} L_{0}(\vec{x}) .
$$

Индексы $j, k$ и прочие могут принимать значение 1 или 2. Т.о., поле температуры вне частиц задается общей формулой

$$
\begin{aligned}
T_{f}(\vec{x}) & =T_{\infty}(\vec{x})+\left[A_{0}^{\mathrm{ext}}(1) L_{0}(\vec{x})+H_{j}^{\mathrm{ext}}(1) L_{j}(\vec{x})+F_{j k}^{\mathrm{ext}}(1)(\vec{x})+\right. \\
& \left.+G_{j k l}^{\mathrm{ext}}(1) L_{j k l}(\vec{x})+D_{j k l m}^{\mathrm{ext}}(1) L_{j k l m}(\vec{x})+\ldots\right]+ \\
& +\left[A_{0}^{\mathrm{ext}}(2) L_{0}(\vec{x}-\vec{r})+H_{j}^{\mathrm{ext}}(2) L_{j}(\vec{x}-\vec{r})+F_{j k}^{\mathrm{ext}}(2)(\vec{x}-\vec{r})+\right. \\
& \left.+G_{j k l}^{\mathrm{ext}}(2) L_{j k l}(\vec{x}-\vec{r})+D_{j k l m}^{\mathrm{ext}}(2) L_{j k l m}(\vec{x}-\vec{r})+\ldots\right],
\end{aligned}
$$

где $T_{\infty}$ определена в (2.6). Коэффициенты $A_{0}^{\operatorname{ext}}(N), H_{j}^{\operatorname{ext}}(N), F_{j k}^{\operatorname{ext}}(N)$ и т.п. имеют тензорную природу и не зависят от $\vec{x}$. По всем повторяющимся коэффициентам выполняется суммирование, в ходе которого они "пробегают" оба своих допустимых значения.

Температурные поля внутри включений записываются аналогично, однако мультиполи модифицируются, дабы не иметь неопределенности в центрах частиц:

$$
\begin{aligned}
T_{p}(N, \vec{y}) & =T_{\infty}\left(\vec{r}_{N}\right)+A_{0}^{\mathrm{int}}(N)+H_{j}^{\mathrm{int}}(N) L_{j}(\vec{y})|\vec{y}|^{2}+F_{j k}^{\mathrm{int}}(N) L_{j k}(\vec{y})|\vec{y}|^{4}+ \\
& +G_{j k l}^{\mathrm{int}}(N) L_{j k l}(\vec{y})|\vec{y}|^{6}+D_{j k l m}^{\mathrm{int}}(N) L_{j k l m}(\vec{y})|\vec{y}|^{8}+\ldots
\end{aligned}
$$

Здесь $r_{N}$ - один из двух радиус-векторов $\vec{r}_{1}=(0,0)$ и $\vec{r}_{2}=(r, 0)$, которые соответствуют центрам инородных включений. Верхние индексы "ext" (external) и "int" (internal) позволяют различать тензоры, относящиеся к температуре вне и внутри частиц.

В принципе, разложения (3.1) и (3.2) по мультиполям можно продолжать и далее. Однако в рамках данной работы мы ограничимся мультиполями четвертого ранга и коэффициентами вида $D_{j k l m}$, соответственно. 
Для произвольной функции вида (3.1) выполнены уравнение (2.1) и условие (2.3), а для любой функции вида (3.2) справедливо (2.2). Тем самым, остается лишь удовлетворить (2.4) и (2.5) подбором подходящих тензорных коэффициентов.

Указанные величины должны быть линейны относительно вектора $\vec{T}$; кроме того, в тензорный базис входит единичный вектор $\vec{b}$, направленный вдоль $O x_{1}$. Опуская верхние индексы, получим, что при любом $N=1,2$ тензорные коэффициенты имеют следующую структуру:

$$
\begin{aligned}
A_{0}(N)= & A A(N) T_{1}, \\
H_{j}(N)= & {[H A(N)-H B(N)] T_{s} b_{s} b_{j}+H B(N) T_{j}, } \\
F_{j k}(N)= & {[F A(N)-2 F B(N)] T_{s} b_{s} b_{j} b_{k}+F B(N)\left(T_{j} b_{k}+T_{k} b_{j}\right), } \\
G_{j k l}(N)= & {[G A(N)-3 G B(N)] T_{s} b_{s} b_{j} b_{k} b_{l}+G B(N)\left(T_{j} b_{k} b_{l}+T_{k} b_{j} b_{l}+T_{l} b_{j} b_{k}\right), } \\
D_{j k l m}(N)= & {[D A(N)-4 D B(N)] T_{s} b_{s} b_{j} b_{k} b_{l} b_{m}+D B(N)\left(T_{j} b_{k} b_{l} b_{m}+T_{k} b_{j} b_{l} b_{m}+\right.} \\
& \left.+T_{l} b_{j} b_{k} b_{m}+T_{m} b_{j} b_{k} b_{l}\right) .
\end{aligned}
$$

Чтобы найти $A A^{\text {ext }}(1), \ldots, D B^{\text {int }}(2)$, выражения (3.1)-(3.3) подставляются в (2.4) и (2.5), а полученные равенства раскладываются в ряд по малому параметру $\varepsilon=a / r$.

\section{2. Асимптотическое разложение искомых величин}

Учет мультиполей четвертого ранга позволяет найти распределение температуры с точностью до $\varepsilon^{5}$ (отметим, что в [14] асимптотические разложения "оборваны" на слагаемых порядка $\left.\varepsilon^{2}\right)$. Итоговое решение задачи таково:

$$
\begin{aligned}
& A A^{\mathrm{ext}}(1) \equiv 0, \quad A A^{\mathrm{int}}(1)=a K_{1}\left[-\varepsilon+K_{1} \varepsilon^{3}+2\left(K_{2}-K_{1}\right) K_{1} \varepsilon^{5}\right] \\
& H A^{\mathrm{ext}}(1)=a^{2} K_{1}\left(1-K_{1} \varepsilon^{2}+K_{1}^{2} \varepsilon^{4}\right), \quad H B^{\mathrm{ext}}(1)=a^{2} K_{1}\left(1+K_{1} \varepsilon^{2}+K_{1}^{2} \varepsilon^{4}\right), \\
& H A^{\mathrm{int}}(1)=2 K_{2}\left(1-K_{1} \varepsilon^{2}+K_{1} K_{2} \varepsilon^{4}\right), \quad H B^{\mathrm{int}}(1)=2 K_{2}\left(1+K_{1} \varepsilon^{2}+K_{1} K_{2} \varepsilon^{4}\right), \\
& F A^{\mathrm{ext}}(1)=a^{3} K_{1}^{2}\left(\varepsilon^{3}-K_{1} \varepsilon^{5}\right), \quad F B^{\mathrm{ext}}(1)=a^{3} K_{1}^{2}\left(-\frac{1}{2} \varepsilon^{3}-\frac{1}{2} K_{1} \varepsilon^{5}\right), \\
& F A^{\mathrm{int}}(1)=\frac{K_{1} K_{2}}{a}\left(2 \varepsilon^{3}-2 K_{1} \varepsilon^{5}\right), \quad F B^{\mathrm{int}}(1)=\frac{K_{1} K_{2}}{a}\left(-\varepsilon^{3}-K_{1} \varepsilon^{5}\right), \\
& G A^{\mathrm{ext}}(1)=-\frac{1}{2} a^{4} K_{1}^{2} \varepsilon^{4}, \quad G B^{\mathrm{ext}}(1)=\frac{1}{6} a^{4} K_{1}^{2} \varepsilon^{4}, \quad G A^{\mathrm{int}}(1)=-\frac{K_{1} K_{2}}{a^{2}} \varepsilon^{4}, \\
& G B^{\mathrm{int}}(1)=\frac{K_{1} K_{2}}{3 a^{2}} \varepsilon^{4}, \quad D A^{\mathrm{ext}}(1)=\frac{1}{6} a^{5} K_{1}^{2} \varepsilon^{5}, \\
& D A^{\text {int }}(1)=\frac{K_{1} K_{2}}{3 a^{3}} \varepsilon^{5}, \quad D B^{\mathrm{ext}}(1)=-\frac{1}{24} a^{5} K_{1}^{2} \varepsilon^{5}, \\
&
\end{aligned}
$$

где введены обозначения

$$
K_{1}=\frac{\kappa_{f}-\kappa_{p}}{\kappa_{f}+\kappa_{p}}, \quad K_{2}=\frac{\kappa_{f}}{\kappa_{f}+\kappa_{p}} .
$$

При $\varepsilon \rightarrow 0$ отсюда получается решение задачи об одиночной круглой частице:

$$
T_{f}(\vec{x})=T_{\infty}(\vec{x})+K_{1} T_{j} x_{j}\left(\frac{a}{|\vec{x}|}\right)^{2}, \quad T_{p}(\vec{x})=T_{0}+2 K_{2} T_{j} x_{j}
$$


Для тензоров, относящихся ко второй частице (неважно, имеют они верхний индекс "ext" или "int"), справедливы соотношения

$$
\begin{aligned}
A_{0}(2) & =-A_{0}(1), & & H_{j}(2)=H_{1}(1), \quad F_{j k}(2)=-F_{j k}(1), \\
G_{j k l}(2) & =G_{j k l}(1), & & D_{j k l m}(2)=-D_{j k l m}(1) .
\end{aligned}
$$

Аналогичные равенства имеют место и при решении пространственной задачи о парном термодинамическом взаимодействии одинаковых частиц [15].

Равенства (3.4), (3.6) завершают решение исходной задачи методом мультипольного разложения.

\section{3. Пример сходимости асимптотических разложений}

Введем полярную систему координат с углом $\theta$ так, что $x_{1}=|\vec{x}| \cos \theta, x_{2}=|\vec{x}| \sin \theta$.

Обозначим через $T_{(K)}^{\prime}$ асимптотическое разложение величины $T^{\prime}$ в задаче о двух частицах с точностью до $\varepsilon^{K}$. Для иллюстрации сходимости этих разложений протабулируем их с некоторым шагом $\Delta \theta$ по углу $\theta$ вдоль концентрических окружностей с центром в начале координат и изучим поведение величины $\max \left|T_{(K+1)}^{\prime}-T_{(K)}^{\prime}\right|$.

Выполним расчеты при таких параметрах: $a=1, \kappa_{p} / \kappa_{f} \in\{2,5,10\}, \varepsilon \in\{1 / 5,1 / 3\}$, $|\vec{T}|=5$. При этом отдельно рассмотрим случаи, в которых $\vec{T}$ сонаправлен с $\vec{r}$ и перпендикулярен ему. Окружности, на которых берутся пробные точки, имеют радиус $a / 2$ (внутри первой частицы), $a$ (на ее границе), $2 a$ и $10 a$ (вне обеих частиц), а шаг изменения полярного угла составляет $\Delta \theta=2 \pi / 200$.

Как показывают результаты вычислений, последовательность $\left\{T_{(K)}^{\prime}\right\}$ фундаментальна: разница между соседними ее членами довольно быстро стремится к нулю при любых комбинациях перечисленных выше параметров. Так, при $\vec{T} \| \vec{r}, \varepsilon=1 / 3, \kappa_{p} / \kappa_{f}=2$ и $|\vec{x}|=2$ значение $\max \left|T_{(2)}^{\prime}-T_{(1)}^{\prime}\right|=4.3 \cdot 10^{-2}, \operatorname{a} \max \left|T_{(5)}^{\prime}-T_{(4)}^{\prime}\right|=2.7 \cdot 10^{-3}$. При $|\vec{x}|=10 a$ величина $\max \left|T_{(5)}^{\prime}-T_{(4)}^{\prime}\right|$, как правило, имеет порядок $10^{-5} \div 10^{-6}$.

Легко объяснить такие свойства $T_{(K)}^{\prime}$, наблюдаемые в расчетах. Во-первых, очевидно, скорость сходимости падает с увеличением $\varepsilon$. Во-вторых, при прочих равных условиях сходимость ускоряется с увеличением $|\vec{x}|$. Это связано с тем, что мультиполи, входящие в разложение $T_{f}$, суть однородные функции отрицательных степеней по $|\vec{x}|$.

\section{4. Решение задачи об одиночной частице методом конечных элементов}

Как было отмечено во Введении, аналитический подход не является панацеей; большую важность приобретают численные методы решения задачи, в первую очередь метод конечных элементов (МКЭ). В свою очередь, для применения МКЭ необходимо уметь оценивать погрешность метода, знать основные факторы, влияющие на нее, а также корректно выбирать форму и размеры расчетной области, параметры рассчетной сетки и задавать условия на границе этой области.

В связи с этим аналитическое решение задачи об одиночной частице (3.5) является эталонным. Оно известно точно и поэтому, сравнивая с ним результаты приближенных расчетов, можно оценить их погрешность.

А. О. Сыромясов, Ю. В. Понкратова, Т. В. Меньшакова. К вопросу о моделировании ... 


\section{1. Общий подход к численному решению задачи}

Сначала предположим, что в среде находится одна частица радиуса $a$ с центром в начале координат. Тогда без ограничения общности можно считать, что градиент температуры на бесконечности направлен вдоль оси $O x_{1}: \vec{T}=\left(T_{1}, 0\right)$. Расчетную область здесь и далее будем выбирать в форме квадрата, симметричного относительно начала координат; сторону квадрата обозначим через $H$ и будем считать большой: $H \gg 2 a$.

Поскольку известно, что $T_{f}(\vec{x}) \rightarrow T_{\infty}(\vec{x})$ при $|\vec{x}| \rightarrow \infty$, а границы выбранного квадрата являются изотермами $T_{\infty}$, то значения $T_{f}$ на левой и правой сторонах квадрата логично считать заранее заданными постоянными:

$$
T_{\text {left }}=\left.T_{\infty}\right|_{x_{1}=-H / 2}, \quad T_{\text {right }}=\left.T_{\infty}\right|_{x_{1}=H / 2} .
$$

Относительная разница этих значений должна приближенно представлять собой невозмущенный градиент температуры:

$$
T_{1} \approx \frac{T_{\text {right }}-T_{\text {left }}}{H} .
$$

На двух горизонтальных сторонах квадрата, которые также расположены далеко от частицы, температура зависит только от $x_{1}$, а в направлении $O x_{2}$ (по нормали к этим сторонам) не изменяется. Соответственно, на них выполнено условие теплоизоляции

$$
\frac{\partial T_{f}}{\partial n}=0
$$

В пакете ANSYS, в котором выполнялись приближенные вычисления с использованием MKЭ, теплоизоляция является граничным условием по умолчанию.

Тепловой контакт между инородной частицей и средой, согласно (2.4), является идеальным - потерь тепла на границе раздела не происходит. Соответствующие ограничения в ANSYS также выполняются по умолчанию.

Итак, при численном расчете граничные условия (2.3) должны быть заменены на (4.1), (4.2) при том, что расчетная область велика по размерам. Условия на поверхности частицы остаются неизменными. Геометрия приближенных граничных условий изображена на рис. 4.1.

Помимо геометрических параметров области, перед численным расчетом необходимо задать и теплопроводности среды и включения. При этом можно воспользоваться следующими упрощениями.

Во-первых, Распределение температуры зависит, фактически, не от $\kappa_{f}$ и $\kappa_{p}$ самих по себе, а от их отношения. В этом можно убедиться, например, по значениям параметров $K_{1}$ и $K_{2}$ в формулах (3.4). Поэтому теплопроводность среды можно считать равной единице и в дальнейших исследованиях варьировать только $\kappa_{p}$.

Во-вторых, из (3.5) следует, что $T_{p}$ не зависит от ее радиуса $a$, а $T_{f}$ зависит от отношения $a /|\vec{x}|$. Поэтому можно считать, что $a=1$.

\section{2. Источники погрешности расчетов и метод ее оценки}

Основных источников погрешности при конечно-элементных расчетах два. Вопервых, это погрешность самого МКЭ, связанная как с качеством сетки, так и с тем, что метод в принципе является приближенным. Во-вторых, это погрешность от замены бесконечной области на конечную. Дело в том, что условия (2.3) и (4.1) с постоянными 


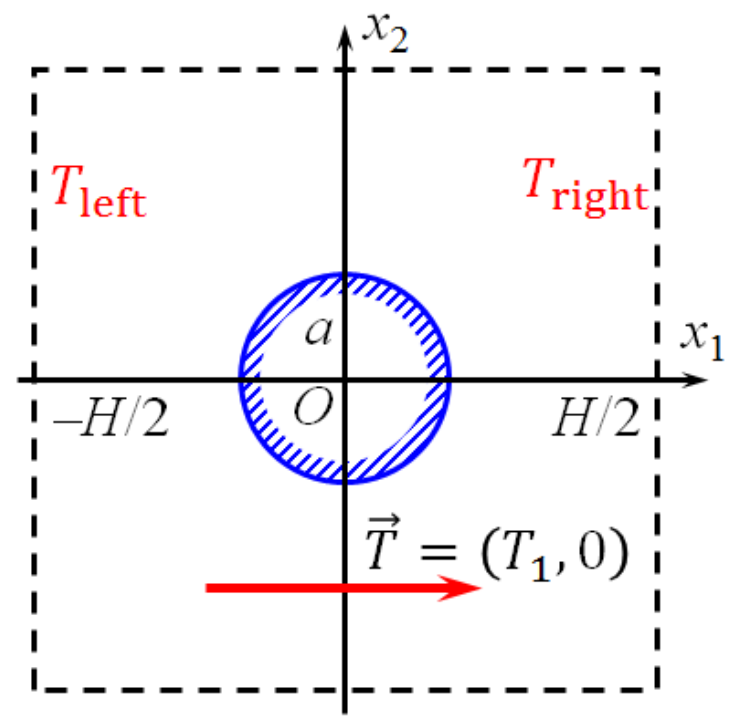

Рис. 4.1. Геометрия приближенных граничных условий

Fig 4.1. Geometry of approximate boundary conditions

$T_{\text {left }}$ и $T_{\text {right }}$, строго говоря, не равносильны: из-за присутствия в среде включения с иной теплопроводностью поле температуры искажается, так что при $x_{1}= \pm H / 2$ равенство $T_{f}=T_{0} \pm T_{1} H / 2$ не выполняется.

Далее установим, какую роль играет каждая из этих погрешностей. Для этого реализуем следующий план действий.

1. Выберем некую расчетную область, на границе которой в качестве граничных условий укажем точные значения $T_{f}(\vec{x})$ из эталонного решения $(3.5)$, например,

$$
T_{\text {right }}=T_{0}+T_{1} \cdot \frac{H}{2} \cdot\left[1+K_{1} \frac{a^{2}}{(H / 2)^{2}+x_{2}^{2}}\right] .
$$

При этом подходе погрешность, связанная с заменой бесконечной области на конечную, исчезает, и можно исследовать погрешность непосредственно МКЭ.

2. При описанных граничных условиях будем пробовать различные варианты улучшений расчетной сетки до тех пор, пока не достигнем приемлемого уровня погрешности вычислений.

3. Сетка, выбранная в итоге, считается оптимальной. Именно ее настройки в дальнейшем используются при увеличении размеров расчетной области и исследовании погрешности, связанной с неточными граничными условиями.

Фактическая цель расчетов - найти упомянутое выше возмущение температуры $T^{\prime}$, поэтому мерой неточности вычислений будем считать относительную погрешность нахождения этой величины:

$$
\delta_{T}=\frac{\left|T_{\text {analytic }}^{\prime}-T_{\text {ANSYS }}^{\prime}\right|}{\left|T_{\text {analytic }}^{\prime}\right|}
$$


где индексы "analytic" и “ANSYS" отвечают значениям $T^{\prime}$, найденным аналитически и приближенно - в пакете ANSYS Workbench.

При фиксированном $\kappa_{f}$ погрешность $\delta_{T}$ может зависеть как от $|\vec{T}|=T_{1}$, так и от $\kappa_{p}$. В дальнейших численных экспериментах будем считать, что $T_{1}=5$, а $\kappa_{p}$ принимает одно из значений $0.1,0.2,0.5,2,5$ и 10. Наиболее интересны варианты, в которых $\kappa_{p} / \kappa_{f}>1$, т.к. они чаще встречаются на практике [5].

Для сравнения приближенного решения с эталонным будем выводить результаты расчетов в ANSYS вдоль концентрических окружностей различного радиуса с центрами в начале координат: эти линии повторяют геометрию инородного включения. Всего рассматривается 5 окружностей: $|\vec{x}|=a / 2,|\vec{x}|=a,|\vec{x}|=2 a,|\vec{x}|=5 a$ и $|\vec{x}|=10 a$. На каждой из них значения $T^{\prime}$ вычисляются в 200 точках, соответствующих полярным углам $\theta$, кратным $2 \pi / 200$.

В качестве интегральной меры погрешности логично вычислять среднее значение $\delta_{T}$ вдоль той или иной окружности. Но при $\theta=\pi / 2$ и $\theta=3 \pi / 2$, т.е. в точках на оси $O x_{2}$, величина $T_{\text {analytic }}^{\prime}=0$, поэтому погрешность становится бесконечной. В точках, близких к $\theta=\pi / 2$ и $\theta=3 \pi / 2$, погрешность также (и по той же причине) очень велика. Чтобы отбросить такие "оторванные" значения $\delta_{T}$, используется критерий вариационного размаха [16]. Итоговой величиной, характеризующей ошибку вычислений, служит $\left\langle\delta_{T}\right\rangle$ - среднее значение $\delta_{T}$ после отбрасывания "оторванных" значений.

\section{3. Погрешность МКЭ}

Глобальное измельчение сетки ресурсоемко, поэтому для улучшения ее свойств используется инструмент Inflation - измельчение шага сетки вблизи линии раздела "среда - частица". Это позволяет более адекватно моделировать температурный пограничный слой, возникающий из-за скачка теплопроводностей. В ходе численных экспериментов было установлено, что оптимальной является сетка с 10 инфляционными слоями, причем каждый следующий слой (считая от границы раздела) должен быть вдвое шире предыдущего. При этом средняя погрешность принимает достаточно малые значения, а производительность вычислений практически не понижается.

Вычисления с точными граничными условиями типа (4.3) велись при $H=20 a$. В итоге были установлены следующие характерные черты поведения $\left\langle\delta_{T}\right\rangle$.

Во-первых, при прочих равных условиях $\left\langle\delta_{T}\right\rangle$ возрастает при увеличении радиуса окружности, вдоль которой выводится решение. Так, при $\kappa_{p}=10$ и $|\vec{x}|=2 a$ значение $\left\langle\delta_{T}\right\rangle=1.50 \cdot 10^{-3}$, а при $|\vec{x}|=10 a$ погрешность составляет $2.22 \cdot 10^{-2}$. Это объясняется тем, что исправление свойств сетки с помощью инструмента Inflation является локальным. Кроме того, при удалении от частицы $T_{\text {analytic }}^{\prime}$ уменьшается, что также увеличивает погрешность.

Во-вторых, $\left\langle\delta_{T}\right\rangle$ увеличивается при приближении $\kappa_{p}$ к 1 . Так, при $|\vec{x}|=5 a$ и $\kappa_{p}=10$ величина $\left\langle\delta_{T}\right\rangle=5.00 \cdot 10^{-3}$, а при $\kappa_{p}=2$ она достигает значения $1.31 \cdot 10^{-2}$. Причиной такого поведения служит то, что при $\kappa_{p} \rightarrow \kappa_{f}$ значение $T_{\text {analytic }}^{\prime} \rightarrow 0$.

Подытоживая, отметим, что погрешность МКЭ “самого по себе" в точках вдали от инородного включения имеет порядок $10^{-2}$, а вблизи него может быть существенно улучшена с помощью инфляции сетки. Описанные параметры этого улучшения далее применяются и к расчетам с неточными граничными условиями типа (4.1).

A. O. Syromyasov, Yu. V. Ponkratova, T. V. Menshakova. On modelling of thermodynamic interaction of... 


\section{4. Погрешность от замены бесконечной области на конечную}

Решая задачу с условиями (4.1), (4.2) на границе расчетной области, будем исследовать зависимость $\left\langle\delta_{T}\right\rangle$ от размера области $H$ и от отношения $\kappa_{p} / \kappa_{f}$. Характерные черты этой зависимости таковы.

Аналогично расчетам с точными граничными условиями, при фиксированных $\kappa_{p}$ и $H$ значение $\left\langle\delta_{T}\right\rangle$ растет с увеличением $|\vec{x}|$.

Далее, при фиксированном $|\vec{x}| \geq a$ (вне частицы) погрешность падает при увеличении $H$. Так, при $\kappa_{p}=10$ и $|\vec{x}|=2 a$ значение $\left\langle\delta_{T}\right\rangle=0.028$ при $H=20$ и 0.004 при $H=80$. Такое поведение связано с тем, что при увеличении $H$ улучшается приближение бесконечной области с помощью конечной при неточных граничных условиях.

Достичь предельного значения $\left\langle\delta_{T}\right\rangle$, т.е. ее величины при $H=20 a$ и точных граничных условиях (ранее эта величина была принята за погрешность собственно МКЭ), не удается. Однако при $H=80 a$ и произвольных $|\vec{x}|$ и $\kappa_{p}$ она имеет приемлемые значения не более 0.1 .

Наконец, при $\kappa_{p} / \kappa_{f}>1$ погрешность растет с убыванием $\kappa_{p}$. Это также объясняется тем, что с уменьшением больших $\kappa_{p}$ и с их приближением к $\kappa_{f}$ величина $T_{\text {analytic }}^{\prime}$ стремится к нулю.

Резюмируя, отметим, что для обеспечения приемлемой точности вычислений $\left(\left\langle\delta_{T}\right\rangle \leq 0.1\right)$ требуется брать размеры расчетной области в $4-5$ раз больше, чем размеры той области, в которой нас интересует распределение температуры. Так, максимальному $|\vec{x}|=10 a$ соответствует $H / 2=40 a$.

\section{5. Численное моделирование парного термодинамического вза- имодействия на плоскости}

Применим полученные выводы к расчету температуры вблизи двух круглых частиц на плоскости. Ограничимся случаем, когда центры включений расположены на расстоянии $r=5 a$ один от другого (см. рис. 2.1), а величина невозмущенного градиента температуры $|\vec{T}|=5$. Температурные пограничные слои вблизи частиц будем учитывать с помощью инфляции сетки с найденными ранее параметрами. Значения $T^{\prime}$ вычислим вдоль концентрических окружностей радиусов $a / 2, a, 2 a$ и $10 a$ (окружность радиуса $5 a$, проходящая как внутри среды, так и внутри частицы 2 , исключена).

По сравнению с задачей об одиночном включении в данном случае возникают следующие дополнительные проблемы.

Необходимо учесть, что градиент температуры $\vec{T}$ может быть по-разному ориентирован относительно $\vec{r}$ и, соответственно, относительно оси $O x_{1}$. В силу линейности исходной задачи достаточно вместо одного расчета с произвольным $\vec{T}=\left(T_{1}, T_{2}\right)$ выполнить два, в которых $\vec{T}=\left(T_{1}, 0\right)$ и $\vec{T}=\left(0, T_{2}\right)$, соответственно.

Кроме того, следует изменить размер расчетной области. Наиболее очевидным решением представляется увеличить сторону квадрата (при прочих равных условиях) с $H$ на $H+2 r$, так что $\left|x_{1,2}\right| \leq H / 2+r$. Возникает вопрос, достаточен ли такой размер области для обеспечения приемлемой погрешности?

Ниже подробно обсуждается вторая из обозначенных проблем. 


\section{1. Проблема определения размеров области в случае двух частиц}

Для более корректной оценки размеров расчетной области сведем задачу о двух частицах к задаче об одиночном включении, которая была рассмотрена выше.

Из (3.4) следует, что в начальном приближении вне обеих идентичных частиц возмущение температуры имеет вид $T^{\prime}(\vec{x})=K_{1} a^{2} T_{j}\left[L_{j}(\vec{x})+L_{j}(\vec{x}-\vec{r})\right]$. Если $|\vec{x}| \gg r$, то $L_{j}(\vec{x}-\vec{r}) \approx L_{j}(\vec{x})$, поэтому на большом расстоянии от обеих частиц

$$
T^{\prime}(\vec{x})=2 K_{1} T_{j} x_{j}\left(\frac{a}{|\vec{x}|}\right)^{2} \text {. }
$$

Сравнивая это выражение с (3.5), получим, что система из двух одинаковых включений при больших $|\vec{x}|$ эквивалентна одному "фиктивному" включению с той же теплопроводностью и радиусом $a \sqrt{2}$. Аналогично можно показать, что облако из $N$ идентичных частиц на плоскости можно приближенно заменить одной частицей радиуса $a \sqrt{N}$.

Отметим, что при выводе этой оценки мы пренебрегли поправками к (3.5), которые, согласно (3.4), имеют порядок $\varepsilon^{2}$. Поэтому дополнительным условием, при котором замена системы инородных включений на единственную частицу будет адекватной, является $a^{2} \ll r^{2}$.

Теперь ответим на следующий вопрос. Пусть радиус включения изменился в $\alpha>1$ раз. Во сколько раз надо изменить размеры квадратной расчетной области для поддержания прежнего значения $\left\langle\delta_{T}\right\rangle$ при тех же $|\vec{T}|$ и $\kappa_{p} / \kappa_{f}$ ?

Для этого вернемся к задаче об одиночной частице при $\vec{T}$, направленном вдоль $O x_{1}$. Напомним, что значения $H=80$ при $T_{1}=5$ и $a=1$ достаточно, чтобы погрешность вычислений была достаточно мала. Поэтому вычислим $\left\langle\delta_{T}\right\rangle$ при $\kappa_{p}=10, T_{1}=5$ вдоль окружностей радиусами $2 a, 5 a$ и $10 a$. При этом радиус включения будем брать равным $\alpha a$, где $\alpha$ принимает значение $1, \sqrt{2}$ и $\sqrt{3}$, а в качестве нового размера области рассмотрим $H, \alpha H$ и $\alpha^{2} H$. В результате численного эксперимента выяснилось, что хотя при увеличении $H$ значение $\left\langle\delta_{T}\right\rangle$ ожидаемо падает, но прежнего $H=80$ даже при увеличенных радиусах частицы достаточно, чтобы обеспечить прежнюю точность. Возможно, это связано с тем, что при росте $а$ увеличивается и $T_{\text {analytic }}^{\prime}$, что приводит к снижению относительной погрешности.

В качестве иллюстрации приведем графики $T_{\mathrm{ANSYS}}^{\prime}$, вычисленных вдоль окружности $|\vec{x}|=20$ внутри расчетной области с одной и той же стороной $H=80$. На рис. 5.1 по оси абсцисс откладываются номера точек на окружности, причем номер 1 соответствует точке с $\theta=0$. Более симметричная синяя линия изображает $T_{\mathrm{ANSYS}}^{\prime}$, рассчитанную для одной частицы радиуса $\sqrt{2}$, а менее симметричная оранжевая - для двух частиц радиуса 1 , расположенных на расстоянии $r=5$ друг от друга.

Как видно, несмотря на некоторые отличия, графики достаточно хорошо накладываются друг на друга, что подтверждает правильность предлагаемого подхода.

Тем самым, нами показано, что при оценке размеров расчетной области $H$ несколько частиц могут быть заменены на одну "фиктивную" большего радиуса. При этом, если прежнее значение $H$ много больше радиуса новой частицы, а расстояние между прежними частицами больше их радиуса, то увеличивать $H$ не имеет смысла.

\section{2. Сходимость асимптотических разложений к численному результату}

Наконец, сравним значения $T^{\prime}$ в задаче о двух включениях, найденные двумя разными способами: методом мультипольных разложений и рассчитанные в пакете ANSYS. 


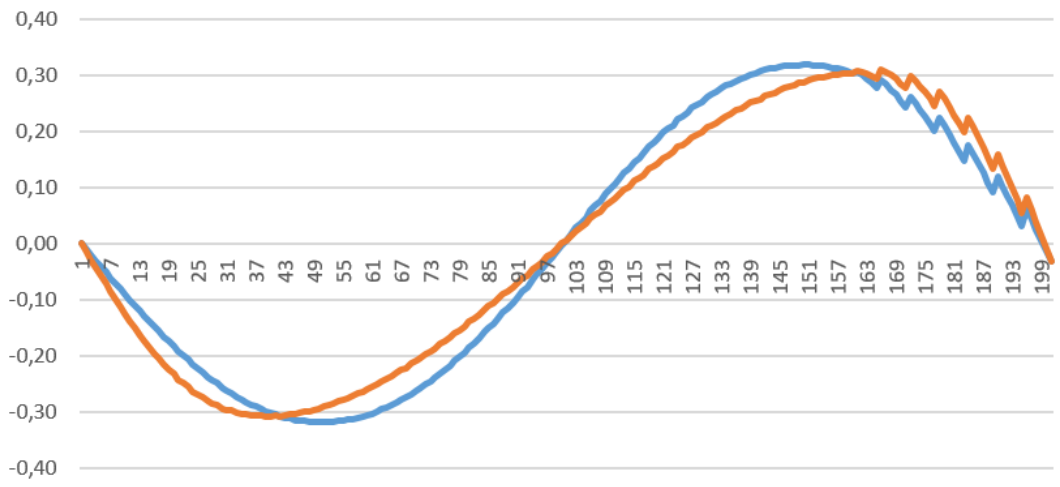

Рис. 5.1. Эффект замены двух частиц одной фиктивной

Fig 5.1. Effect of replacement of two particles by single "fictituous" one

Для этого воспользуемся результатами раздела 3.3. Будем сравнивать $T_{(K)}^{\prime}$ с величи-

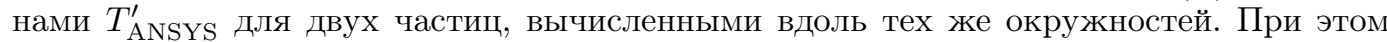
ограничимся значением $\varepsilon=1 / 5$. Сторону $H$ квадрата, включающего обе частицы, возьмем равной 90: к найденному ранее значению $H=80$ прибавим удвоенное расстояние между центрами частиц.

Находя $\max \left|T_{(5)}^{\prime}-T_{\mathrm{ANSYS}}^{\prime}\right|$, получим, что разница между аналитическим и численным решениями невелика: при всех сочетаниях испытуемых параметров она имеет порядок $10^{-2}$. Тем самым, можно утверждать, что оба подхода к расчету поля температуры дают сходные результаты, а следовательно, оба они корректны. С другой стороны, $\max \left|T_{(5)}^{\prime}-T_{(4)}^{\prime}\right|$ принимает гораздо меньшие значения - порядка $10^{-5}$. Это позволяет считать асимптотическое разложение практически точным при заданных параметрах.

\section{6. Заключение}

В статье исследована модельная задача о парном термодинамическом взаимодействии одинаковых круглых частиц на плоскости в присутствии постоянного на бесконечности градиента температуры. Целью исследования является сравнение двух подходов к решению аналогичных задач. Первый из этих подходов - аналитический, основанный на мультипольном представлении искомых функций и асимптотическом разложении коэффициентов этого представления. Второй подход, численный, использует в качестве инструмента МКЭ.

С помощью аналитического метода разложение полей температуры вне и внутри включений было найдено с точностью до $\varepsilon^{5}$, где $\varepsilon<1$ - это безразмерный радиус частиц. На конкретном примере было показано, что последовательность асимптотических приближений является фундаментальной и сходится достаточно быстро.

При отыскании распределения температуры с помощью МКЭ был решен ряд подзадач. Во-первых, были выявлены источники ошибок метода, в частности, замена бесконечной области на конечную и, как следствие, неточность условий на границе области. Во-вторых, для задачи об одиночной частице была предложена методика оценки погрешности в зависимости от геометрических и физических параметров задачи, а также управления этой погрешностью. В-третьих, в задаче о нескольких частицах предложен 
способ оценки параметров расчетной области с помощью замены исходных частиц на одну, имеющую ту же теплопроводность, но больший радиус. Была показана эффективность такой замены на значительных расстояниях от центров инородных включений.

Наконец, был проведено сравнение возмущений, вносимых частицами в распределение температуры, рассчитанных разными методами. В итоге было получено, что оба подхода дают близкие результаты, что подтверждает их корректность.

\section{СПИСОК ЛИТЕРАТУРЫ}

1. Бердичевский В. Л. Вариационные принципы механики сплошной среды. М.: Наука. Гл. ред. физ.-мат. лит., 1983. 448 с.

2. Александров В. Ю. Сопротивление сильно нагретой сферы при малых числах Рейнольдса // Известия РАН. Механика жидкости и газа. 2011. № 5. С. 142-156.

3. Padrino J. C., Sprittles J. E., Lockerby J. E. Thermophoresis of a spherical particle: modelling through moment-based, macroscopic transport equations // Journal of Fluid Mechanics. 2019. Vol. 862. P. 312-347. DOI: https://doi.org/10.1017/jfm.2018.907

4. Глушак А. В., Малай Н. В., Миронова Н. Н. Решение краевой задачи для линеаризованных по скорости уравнений Навье - Стокса в случае неизотермического обтекания нагретого сфероида газообразной средой // Журнал вычислительной математики и математической физики. 2012. Т. 52. № 5. С. 946-959.

5. Глушак А. В., Малай Н. В., Щукин Е. Р. Решение краевой задачи для линеаризованных по скорости уравнений Навье - Стокса в случае седиментации нагретой твердой гидрозольной частицы сферической формы // Журнал вычислительной математики и математической физики. 2018. Т. 58. № 7. С. 1178-1188.

6. Batchelor G. K., Green J. T. The hydrodynamic interaction of two small freely-moving spheres in a linear flow field // Journal of Fluid Mechanics. 1972. Vol. 56. Pt. 2. P. 375-400. DOI: https://doi.org/10.1017/S0022112072002927

7. Batchelor G. K., Green J. T. The determination of the bulk stress in a suspension of spherical particles to order $c^{2} / /$ Journal of Fluid Mechanics. 1972. Vol. 56. Pt. 3. P. 401-427. DOI: https://doi.org/10.1017/S0022112072002435

8. Мартынов С. И. Гидродинамическое взаимодействие частиц // Известия РАН. Механика жидкости и газа. 1998. № 2. С. 112-119.

9. Ландау Л. Д., Лифшиц Е. М. Теоретическая физика. Т. VI. Гидродинамика. М.: Наука. Гл. ред. физ.-мат. лит., 1986. 736 с.

10. Ландау Л. Д., Лифшиц Е. М. Теоретическая физика. Электродинамика сплошных сред. М.: Гос. изд-во физ.-мат. лит., 1959. 532 с.

11. Хаппель Дж., Бреннер Г. Гидродинамика при малых числах Рейнольдса. М.: Мир, 1976. $632 \mathrm{c}$.

12. Де Брейн Н. Асимптотические методы в анализе. М.: Иностранная литература, 1961. 248 c. 
13. Сегерлинд Л. Применение метода конечных элементов. М.: Мир, 1979. 392 с.

14. Меньшакова Т. В., Сыромясов А. О. Термодинамическое взаимодействие двух круглых частиц на плоскости // Математическое и компьютерное моделирование естественно-научных и социальных проблем. Материалы XIII Международной научно-технической конференции молодых специалистов, аспирантов и студентов. - Пенза, 2019. - С. 167-171.

15. Сыромясов А. О. Термодинамическое взаимодействие сферических частиц в среде с постоянным градиентом температуры // Вестник Нижегородского университета им. Н. И. Лобачевского. 2011. № 4, ч. 3. С. 1158-1160.

16. Третьяк Л. Н. Обработка результатов наблюдений. Оренбург.: ГОУ ОГУ, 2004. 171 c.

Поступила 03.09.2021; доработана после рещензирования 28.10.2021; принята к публикации 16.11.2021

Информаиия об авторах:

Сыромясов Алексей Олегович, доцент кафедры прикладной математики, дифференциальных уравнений и теоретической механики, Национальный исследовательский Мордовский государственный университет (430005, Россия, г. Саранск, ул. Большевистская, д. 68/1), кандидат физико-математических наук, ORCID: http://orcid.org/0000-0001-6520-0204, syal1@yandex.ru

Понкратова Юлия Владимировна, магистрант кафедры прикладной математики, дифференциальных уравнений и теоретической механики, Национальный исследовательский Мордовский государственный университет (430005, Россия, г. Саранск, ул. Большевистская, д. 68/1), ORCID: http://orcid.org/0000-0002-0741-1262, ponkratova-yuliya@mail.ru

Меньшакова Татьяна Вячеславовна, аспирант кафедры прикладной математики, дифференциальных уравнений и теоретической механики, Национальный исследовательский Мордовский государственный университет (430005, Россия, г. Саранск, ул. Большевистская, д. 68/1), ORCID: http://orcid.org/0000-0002-7728-7674, menshakova_94@mail.ru

Авторы прочитали и одобрили окончательный вариант рукописи.

Конфликт интересов: авторы заявляют об отсутствии конфликта интересов.

А. О. Сыромясов, Ю. В. Понкратова, Т. В. Меньшакова. К вопросу о моделировании ... 


\title{
On modelling of thermodynamic interaction of particles suspended in two-dimensional medium
}

\author{
A. O. Syromyasov, Yu. V. Ponkratova, T. V. Menshakova \\ Ogarev Mordovia State University (Saransk, Russian Federation)
}

\begin{abstract}
Analytical description of temperature distribution in a medium with foreign inclusions is difficult due to the complicated geometry of the problem, so asymptotic and numerical methods are usually used to model thermodynamic processes in heterogeneous media. To be convinced in convergence of these methods the authors consider model problem about two identical round particles in infinite planar medium with temperature gradient which is constant at infinity. Authors refine multipole expansion of the solution obtained earlier by continuing it up to higher powers of small parameter, that is nondimensional radius of thermodynamically interacting particles. Numerical approach to the problem using ANSYS software is described; in particular, appropriate choice of approximate boundary conditions is discussed. Authors ascertain that replacement of infinite medium by finite-sized domain is important source of error in FEM. To find domain boundaries in multiple inclusions' problem the authors develop "fictituous particle" method; according to it the cloud of particles far from the center of the cloud acts approximately as a single equivalent particle of greater size and so may be replaced by it. Basing on particular quantitative data the dependence of domain size that provides acceptable accuracy on thermal conductivities of medium and of particles is explored. Authors establish series of numerical experiments confirming convergence of multipole expansions method and FEM as well; proximity of their results is illustrated, too.
\end{abstract}

Key Words: thermodynamic interaction, disperse medium, heat equation, multipole, asymptotic expansion, finite element method

For citation: A. O. Syromyasov, Yu. V. Ponkratova, T. V. Menshakova. On modelling of thermodynamic interaction of particles suspended in two-dimensional medium. Zhurnal Srednevolzhskogo matematicheskogo obshchestva. 23:4(2021), $444-460$. DOI: https://doi.org/10.15507/2079-6900.23.202104.444-460

\section{REFERENCES}

1. V.L. Berdichevsky, /Variational principles of continuum mechanics/, Nauka, Moscow, 1983 (In Russ.), 448 p.

2. V. Yu. Alexandrov, "Drag of a strongly heated sphere at small Reynolds numbers", Fluid Dynamics, 46:5 (2011). DOI: https://doi.org/10.1134/S0015462811050139

3. J. C. Padrino, J. E. Sprittles, J. E. Lockerby, "Thermophoresis of a spherical particle: modelling through moment-based, macroscopic transport equations", Journal of Fluid Mechanics, 862 (2019), 312-347. DOI: https://doi.org/10.1017/jfm.2018.907

4. A. V. Glushak, N. V. Malay, N. N. Mironova, "[Boundary value problem for the Navier-Stokes equations linearized in rate in the case of non-isothermal flow of heated gaseous medium spheroid]", Zh. Vychisl. Mat. Mat. Fiz., 52:5 (2012), 946-959 (In Russ.).

5. N. V. Malai, A. V. Glushak, E. R. Shchukin, "Solution of a Boundary Value Problem for Velocity-Linearized Navier-Stokes Equations in the Case of a Heated Spherical Solid 
Particle Settling in Fluid", Computational Mathematics and Mathematical Physics, 58:7 (2018), 1132-1141. DOI: https://doi.org/10.1134/S0965542518070114

6. G.K. Batchelor, J.T. Green, "The hydrodynamic interaction of two small freelymoving spheres in a linear flow field", Journal of Fluid Mechanics, 56:2 (1972), 375400. DOI: https://doi.org/10.1017/S0022112072002927

7. G. K. Batchelor, J. T. Green, "The determination of the bulk stress in a suspension of spherical particles to order $c^{2 "}$, Journal of Fluid Mechanics, 56:3 (1972), 401-427. DOI: https://doi.org/10.1017/S0022112072002435

8. S. I. Martynov, "Hydrodynamic interaction of particles", Fluid Dynamics, 33:2 (1998), 245-251. DOI: https://doi.org/10.1007/BF02698709

9. L. D. Landau, E. M. Lifshitz, Fluid Mechanics, Butterworth-Heinemann, 1987, 539 p.

10. L. D. Landau, E. M. Lifshitz, Electrodynamics of Continuous Media, Pergamon Press, 1960, 417 p.

11. J. Happel, H. Brenner, Low Reynolds number hydrodynamics, Springer Netherlands, 1983, xii, 553 p.

12. N. de Bruijn, Asymptotic methods in analysis, North-Holland, Amsterdam, 1958.

13. L. J. Segerlind, Applied Finite Element Analysis, John Wiley and Sons, New York / London / Sydney / Toronto, 1976.

14. T. V. Menshakova, A. O. Syromyasov, "[Thermodynamic interaction of two round particles on a plane]", Mathematical and Computer Modelling of Natural Science and Social Problems (MCM-2019), Proc. XIII Int. Scientific-Technical Conference of young scientists, PhD students and students (Penza, 3-6 June, 2019), 167-171.

15. A. O. Syromyasov, "[Thermodynamic interaction of spherical particles in a medium with constant temperature gradient]", Vestnik of Lobachevsky University of Nizhni Novgorod, 2011, no. 4, 1158-1160 (In Russ.).

16. L. N. Tretyak, [Processing of observation results], Orenburg State University, 2004 (in Russ.), $171 \mathrm{p}$.

Submitted 03.09.2021; Revised 28.10.2021; Accepted 16.11.2021

Information about the authors:

Aleksei O. Syromyasov, Associate Professor, Department of Applied Mathematics, Differential Equations and Theoretical Mechanics, Ogarev Mordovia State University (68/1, Bolshevistskaya str., Saransk 430005, Russia), Ph.D (Physics and Mathematics), ORCID: http://orcid.org/0000-0001-6520-0204, syal1@yandex.ru

Yulia V. Ponkratova, Master student, Department of Applied Mathematics, Differential Equations and Theoretical Mechanics, Ogarev Mordovia State University (68/1, Bolshevistskaya str., Saransk 430005, Russia), ORCID: http://orcid.org/0000-0002-0741-1262, ponkratovayuliya@mail.ru

Tatyana V. Menshakova, Ph.D. student, Department of Applied Mathematics, Differential Equations and Theoretical Mechanics, Ogarev Mordovia State University (68/1, 
Bolshevistskaya str., Saransk 430005, Russia), ORCID: http://orcid.org/0000-0002-7728-7674, menshakova_94@mail.ru

The authors have read and approved the final manuscript.

Conflict of interest: The authors declare no conflict of interest. 